\title{
¿ES SEGURO PARA LA SALUD HUMANA Y ANIMAL EL USO DE RATICIDAS QUE CONTIENEN SALMONELLA?
}

\author{
Alberto Mendoza ${ }^{1, a}$, Víctor Chávez ${ }^{1, a}$, Gisely Hijar ${ }^{1, b}$, Jessica Loyola1,c
}

\begin{abstract}
RESUMEN
Se realizó una búsqueda sistemática para identificar publicaciones sobre la seguridad ante la exposición de raticidas que contienen Salmonella para humanos y animales. Se consideraron publicaciones a texto completo que incluían descripción de su metodología y la presentación adecuada de sus resultados. De 545 publicaciones recuperadas, 47 se revisaron a texto completo de las que se seleccionaron 12. En seis se reportan casos de salmonelosis en humanos, incluso casos fatales, asociados a la exposición a versiones anteriores de estos raticidas. El único ensayo clínico encontrado reporta una mayor frecuencia de diarrea y fiebre en el grupo que ingirió Salmonella contenida en Biorat (presentación $^{\circ}$ comercial actual); sin embargo, la diferencia no fue estadísticamente significativa, pero el ensayo presentó problemas metodológicos. Las cepas de Salmonella enteritidis contenidas en una versión anterior (Ratin®) y en la versión actual corresponden a la misma variedad (Danysz) y fagotipo (6a), y están cercanamente relacionadas según la técnica de electroforesis en gel de campo pulsado (PFGE). No se reporta efectos patógenos de esta Salmonella para las diferentes especies de animales ensayadas; sin embargo, se encontraron limitaciones en la metodología empleada. Se concluye que la Salmonella enteritidis contenida en versiones anteriores de raticidas produjo enfermedad en humanos por lo que fue prohibida su comercialización y que existiría un riesgo potencial de la versión actual por contener una bacteria muy similar y por no tener evidencia suficiente que garantice su seguridad. Son necesarios estudios bien diseñados por instituciones sin conflicto de interés, antes de su aplicación en salud pública o agricultura.
\end{abstract}

Palabras clave: Rodenticidas; Salmonella; Salmonella enteritidis; Salud pública; Seguridad (Fuente: DeCS BIREME)..

\section{IS SAFETY FOR HUMAN AND ANIMAL HEALTH THE USE OF SALMONELLA-BASED RODENTICIDES}

\begin{abstract}
We conducted a systematic search of the literature to identify publications on the safety of exposure to Salmonella-based rodenticides by humans and animals. We included full-text publications that described the methods and presented their results satisfactorily. Of 545 publications retrieved, 47 were reviewed in full text and from those 12 were selected. Six reports featured cases of salmonellosis in humans, with fatal cases, associated with exposure to previous versions of this type of rodenticide. A clinical trial reported an increased frequency of diarrhea and fever in the group that ingested Biorat ® (the current commercial form) containing Salmonella, however the difference from the control group was not significant, but the trial had methodological problems. Strains of Salmonella enteritidis from an earlier version of the rat poison (Ratin $\left.{ }^{\circledR}\right)$ and those in the current version correspond to the same variety (Danyzs) and phage type (6a), and were found to be closely related using the technique of pulsed field gel electrophoresis (PFGE). No pathogenic effects of this Salmonella were reported in different animal species tested; however, we found limitations in the methodology. We conclude that the Salmonella enteritidis contained in earlier rat poison formulations produced illness in humans so that its commercialization was prohibited, and that there would be a potential risk with the present formulation because it contains a very similar bacteria, and because there is not sufficient evidence to guarantee its safety. Well-designed studies still need to be done by institutions that do not have a conflict of interest before it can be applied in the areas of public health and agriculture.
\end{abstract}

Key words: Rodenticidas, Salmonella, Salmonella enteritidis; Public health; Safety (source: MeSH NLM).

\section{INTRODUCCIÓN}

El uso de raticidas biológicos como medida de intervención para el control de roedores genera controversia sobre el riesgo de su potencial exposición accidental o intencional a seres humanos y otros animales ${ }^{(1-3)}$. La Organización Mundial de la Salud (OMS) enfatiza que las especies de Salmonella no deben ser usadas como raticidas por el riesgo para la salud pública ${ }^{(4,5)}$, debido a que son patógenas para el ser humano, por ello, los países desarrollados han prohibido su comercialización desde el siglo pasado ${ }^{(1,6)}$.

Sin embargo, versiones recientes de estos productos que contienen 4-hidroxicumarina al 0,02\% y Salmonella enteritidis variedad Danysz, fagotipo $6 a$, lisina negativa, vienen siendo comercializadas en algunos países en desarrollo. De acuerdo con el fabricante, esta Salmonella enteritidis tiene virulencia específica contra ratas y ratones y es totalmente inocua para el hombre y otros animales $(7,8)$.

\footnotetext{
Unidad de Análisis y Generación de Evidencias en Salud Pública (UNAGESP), Centro Nacional de Salud Pública, Instituto Nacional de Salud, Lima, Perú. a Médico Infectólogo; ' Bióloga, Magister en Bioquímica y Biología Molecular; ' Bibliotecóloga.
} 
Para conocer la información existente sobre la seguridad de la exposición a raticidas que contienen Salmonella en el ser humano y animales, se realizó una búsqueda sistemática para identificar publicaciones referentes a la transmisión, infección o desarrollo de manifestaciones clínicas en seres humanos o animales expuestos experimental o accidentalmente a los raticidas que contienen Salmonella enteritidis.

\section{PROCEDIMIENTOS}

La búsqueda de información se hizo de manera sistemática en las siguientes bases bibliográficas: National Library of Medicine (Pubmed/MEDLINE), Cochrane Library, Excerpta Medica Database (EMBASE), Scientific Electronic Library Online (SciELO), Literatura Latinoamericana y del Caribe en Ciencias de la Salud (LILACS), Bibliotecas de la OMS y de la Organización Panamericana de la Salud (OPS), Google Scholar (versión en inglés) y Cybertesis. Se realizó la búsqueda en cada una de las bases bibliográficas y se combinó los resultados para eliminar duplicidades usando el programa EndNote X3.

Se empleó la siguiente estrategia de búsqueda: [Biorat OR Ratin OR Salmocumarin OR (Salmonella AND rodenticides)], limitada a los idiomas inglés, español, portugués y francés; sin limitación por el periodo de publicación. En Google Scholar la búsqueda se limitó a: (Biology, Life Sciences, and Environmental Science) y (Medicine, Pharmacology, and Veterinary Science). Debido a la poca información identificada como trabajos originales se decidió incluir información relevante contenida en artículos de revisión, comunicaciones cortas, cartas al editor, tesis de grados y reportes técnicos del fabricante (expediente o dossier de Biorat $₫$ ). Se excluyó publicaciones que no tuviesen el artículo disponible, publicaciones con aseveraciones sin referencias primarias y artículos periodísticos o publicitarios. Se incluyeron estudios adicionales de las referencias de los artículos a texto completos revisados, como búsqueda secundaria.

Dos autores de manera independiente, seleccionaron los artículos que, de acuerdo al título y resumen, estaban relacionados con el tema de estudio. En caso de discordancia, la decisión de un tercer autor tenía una función dirimente. Se revisó las versiones a texto completo de los artículos que reunían los criterios de inclusión y exclusión, luego tres investigadores evaluaron la calidad de las publicaciones -conclusiones del estudio sean coherentes con los resultados y con los métodos descritos-, aquellos que no cumplían este criterio fueron excluidos. Finalmente, los estudios seleccionados fueron resumidos empleando una matriz de extracción de datos previamente estandarizada, donde se extrajo información correspondiente a país, revista, año de publicación, tipo de publicación, instituciones que realizaron el estudio, declaración de conflicto de interés, población de estudio, objetivos, diseño del estudio, intervención, resultados primarios y secundarios, conclusiones, fortalezas y limitaciones de cada estudio. Para la síntesis final de los resultados, los estudios se estratificaron de acuerdo con la evaluación de la seguridad de los raticidas para humanos y animales, de manera independiente.

\section{HALLAZGOS}

Recuperamos 545 publicaciones: 31 en Pubmed/MEDLINE, siete en EMBASE, tres en LILACS, una en SciELO, una en Cybertesis, 502 en Google Scholar y ninguna en las bibliotecas Cochrane, OMS y OPS. Luego de la revisión de títulos, resúmenes y eliminación de duplicidades, se obtuvieron 37 artículos y 10 referencias secundarias relacionadas con la seguridad de raticidas basados en Salmonella, que fueron revisados a texto completo. Luego de evaluar las 47 referencias seleccionadas, se excluyó 35: 21 por no responder a la pregunta de investigación ${ }^{(9-28)}$, siete por no superar el criterio de calidad planteado en cuanto a sus conclusiones, resultados y métodos usados ${ }^{(4,6,8,29-32)}$, cuatro por no contar con texto completo ${ }^{(33-36)}$ y tres por idioma ${ }^{(37-39)}$. De las 12 publicaciones incluidas para revisión, seis correspondieron a seguridad en humanos ${ }^{(1,2,40-43)}$ y seis en animales ${ }^{(44-49)}$. El proceso de selección se muestra en la Figura 1.

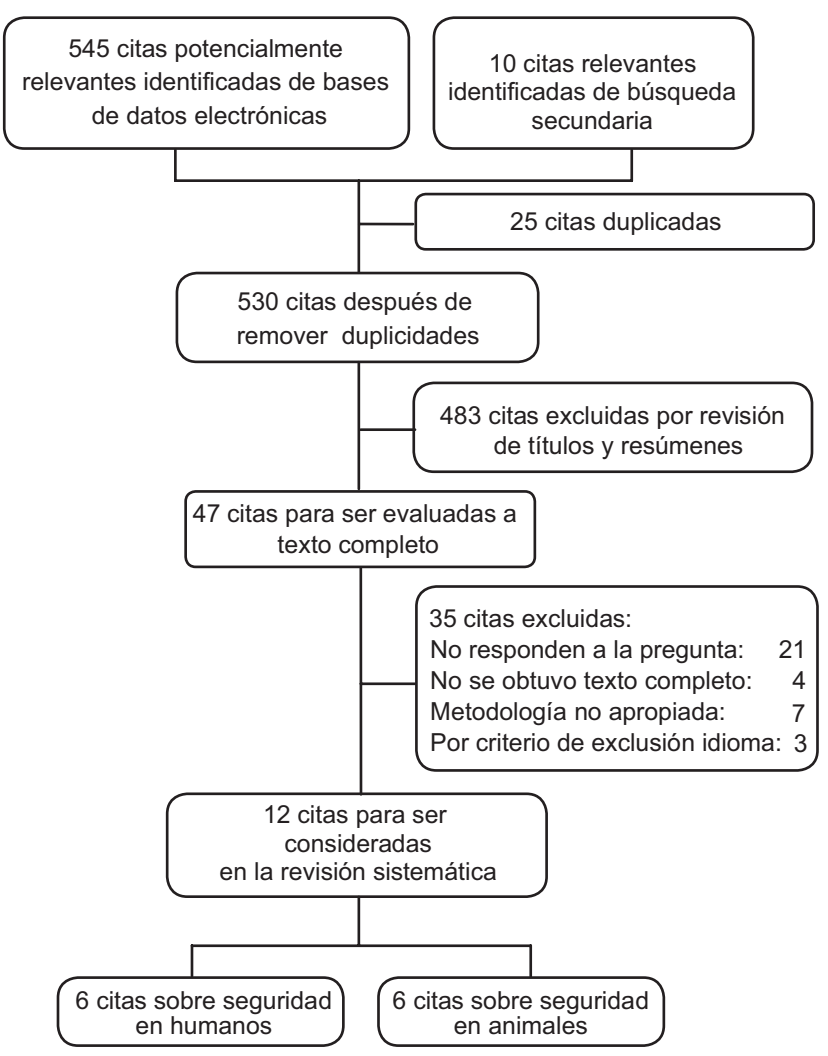

Figura 1. Proceso de selección de artículos incluidos en el análisis. 


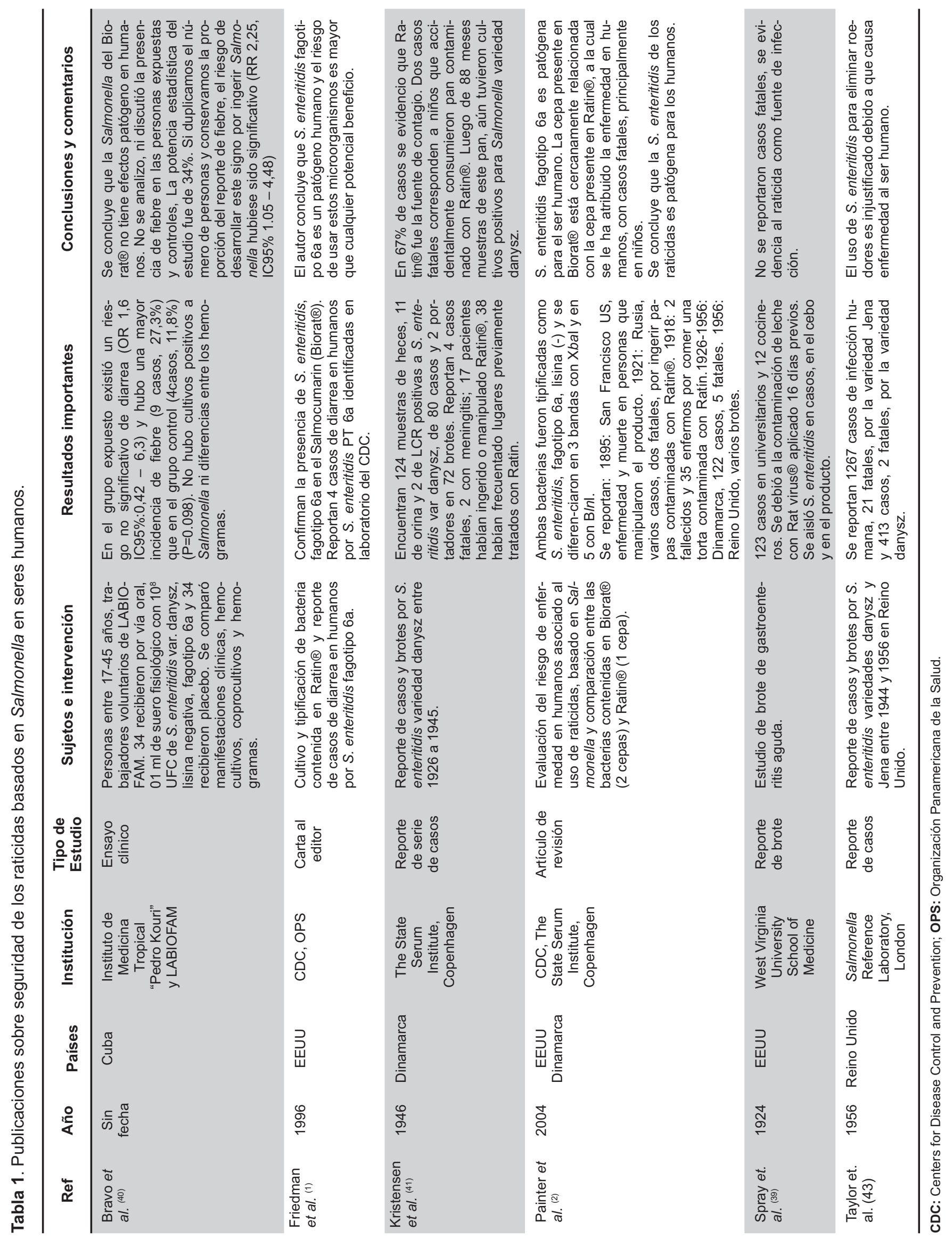




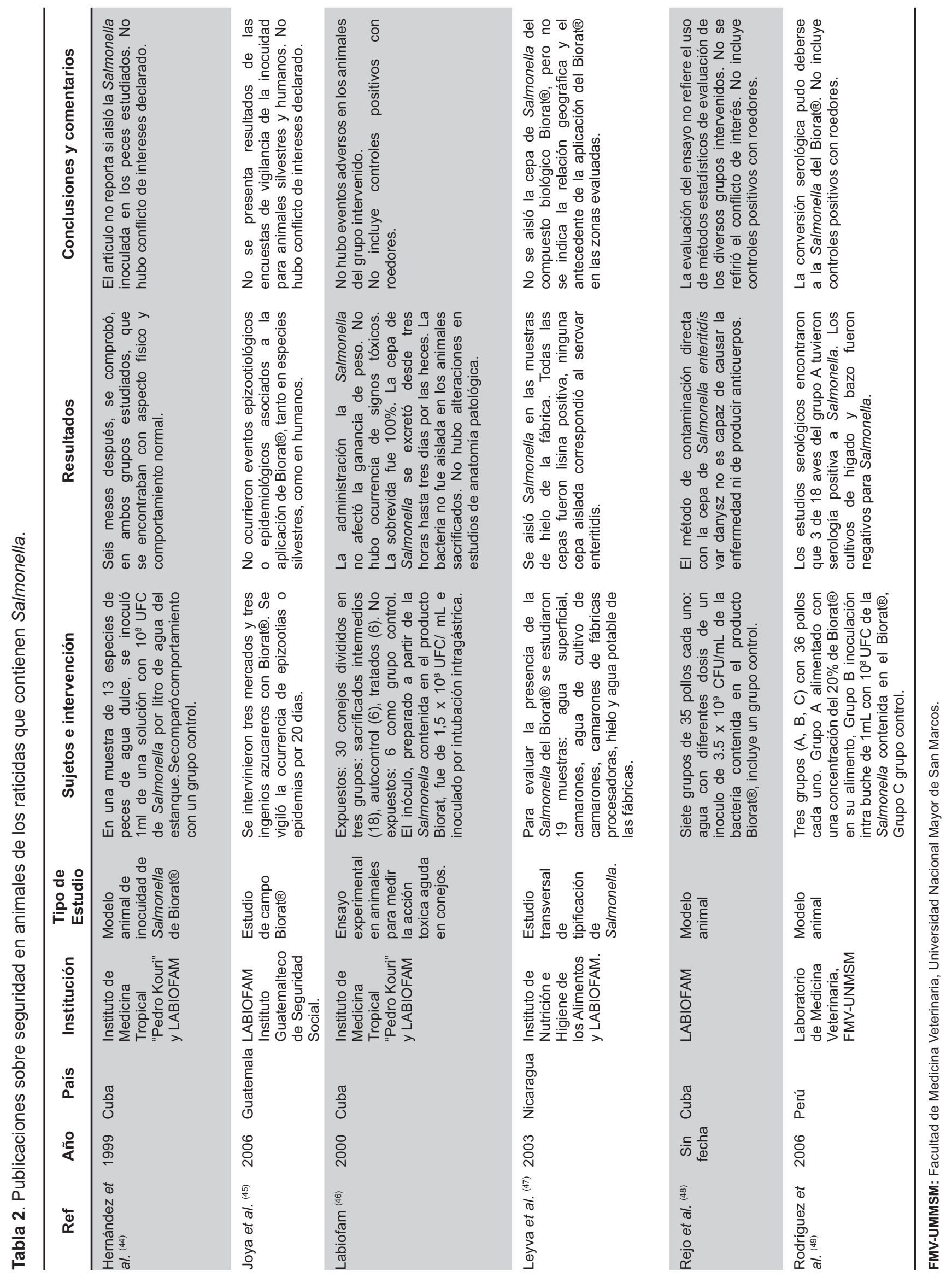




\section{SEGURIDAD EN HUMANOS}

Las seis publicaciones sobre seguridad en humanos (Tabla 1), comprendieron: un ensayo clínico, tres reportes de caso, una carta al editor y una comunicación corta de un brote, todos causados por Salmonella enteritidis variedad Danysz fagotipo 6a. En estas publicaciones se reporta alrededor de 714 casos de gastroenteritis aguda, dos meningitis, nueve casos fatales y otros brotes no cuantificados, causados por esta especie y variedad de Salmonella. La mayoría de estos reportes procedieron de la vigilancia de brotes de gastroenteritis aguda, por laboratorios de referencia nacional en Dinamarca (41), Reino Unido ${ }^{(43)}$ y Estados Unidos ${ }^{(1,2)}$. En más de la tercera parte de los casos reportados se pudo determinar el antecedente de consumo, manipulación o haber estado en lugares previamente tratados con raticidas biológicos, principalmente Ratin $₫$ y Rat-virus $₫$, presentaciones actualmente prohibidas.

Estudios del Centro de Control y Prevención de Enfermedades de Estados Unidos (CDC) ${ }^{(2)}$ y en Reino Unido ${ }^{(3)}$ demuestran que la cepa de Salmonella enteritidis contenida en Ratin ${ }^{\circledR}$ es muy similar a la contenida en el raticida biológico Biorat ${ }^{\circledR}$ (actualmente comercializado en América Latina y Asia). Ambas cepas de Salmonella enteritidis son de la misma variedad (Danysz), del mismo fagotipo 6a, no descarboxilan lisina, y tienen el mismo perfil de tipificación por plásmidos. Por otro lado, por la técnica molecular de electroforesis en gel de campo pulsado (PFGE), ambas cepas de Salmonella enteritidis están cercanamente relacionadas ${ }^{(50)}$, e involucra un ancestro común y no debe ser interpretada como cepas diferentes desde el punto de vista molecular, como lo señalan los fabricantes ${ }^{(30)}$. Sin embargo, se desconoce la implicancia de esta diferencia menor en la patogenicidad y virulencia de las especies de Salmonella ${ }^{(2)}$.

Un ensayo clínico comparó las manifestaciones clínicas y los resultados de los coprocultivos y hemocultivos entre 34 trabajadores de Labiofam (fabricante de Biorat $₫$ ) que ingirieron una solución con $10^{8}$ UFC de Salmonella presente en Biorat $\circledast$ y 34 controles sanos que ingirieron placebo. Seis $(17,6 \%)$ de las personas intervenidas y cuatro $(11,8 \%)(p=0,32)$ de los controles presentaron diarrea aguda $(p=0,32)$. Nueve $(27,3 \%)$ del grupo intervenido y cuatro $(11,8 \%)(p=0,099)$ del grupo control presentaron algún grado de fiebre $(p=0,099)$. No se aisló $S$. enteritidis de las muestras de sangre o heces, ni hubo diferencia en los hemogramas evaluados. Los autores concluyen que la ingesta de la bacteria contenida en Biorat $\AA$ no tiene un efecto patógeno en humanos. Sin embargo, al analizar la potencia del ensayo clínico, calculada con el tamaño de la muestra, esta fue muy baja (34\%) y solo duplicando la población estudiada, el riesgo relativo de desarrollar fiebre por ingerir Salmonella logra ser estadísticamente significativo (RR: 2,25; IC95\%: 1,05-4,8). Tampoco se discute las causas de fiebre en el grupo control, que es un hallazgo infrecuente. Por otro lado, debe destacarse el conflicto de interés de los autores por la necesidad de demostrar la inocuidad de esta Salmonella en el ser humano, porque constituye una fuente de divisas importante según se lee en la justificación de su reporte ${ }^{(40)}$

\section{SEGURIDAD EN ANIMALES}

De las seis publicaciones seleccionadas concernientes a seguridad en animales, todas fueron realizadas exclusivamente por Labiofam o con la coautoría de instituciones de salud o universidades de Centro América y Perú. En cuatro estudios se expuso experimentalmente a peces de río, pollos parrilleros, gallinas y conejos, a concentraciones de aislamientos de Salmonella enteritidis contenida en Biorat $®$, se evaluó el efecto sobre estos animales comparándolos con grupo control no expuesto a la bacteria. La conclusión fue la misma en todas estas experiencias indicando que esta bacteria no es patógena para estas especies ${ }^{(44-47)}$. Sin embargo, en casi todos los experimentos, no se empleó Biorat $\AA$ como producto de ensayo, sino solo el componente bacteriano, no incluyeron el anticoagulante 4-hidroxicumarina contenida en el producto que podría cumplir una función importante en su mecanismo de acción. Tampoco se consideraron controles positivos, es decir, roedores que a las mismas concentraciones ensayadas con otras especies de animales, sean afectados por esta Salmonella, y así demostrar que las dosis ensayadas fueron las convenientes. Otro estudio en Nicaragua demostró que Biorat $($ no estuvo implicado en la contaminación de cultivos de camarones por Salmonella, debido a que no se encontró Salmonella lisina negativa en los aislamientos microbianos de diferentes muestras ${ }^{(48)}$. Finalmente, un estudio en campo en Guatemala no encontró eventos epizoóticos o epidemiológicos hasta 20 días después de la aplicación de Biorat ${ }^{\circledR}{ }^{(49)}$. El resumen de los estudios en animales y sus comentarios se muestran en la Tabla 2. Con la información identificada no se puede establecer conclusiones definitivas en cuanto a la seguridad de estos raticidas.

\section{DISCUSIÓN}

Mediante una búsqueda sistemática de la literatura se ha encontrado evidencia de afección humana por la exposición a Salmonella enteritidis fagotipo 6a, lisina negativa, variedad Danysz contenida en raticidas biológicos. Los reportes corresponden principalmente a brotes de gastroenteritis aguda detectados por los sistemas de vi- 
gilancia en países desarrollados y estuvieron asociados con versiones de raticidas que contienen Salmonella y que han sido retirados del mercado, como Ratin $®$ y Ratvirus $\circledast$. Sin embargo, el producto Biorat $\circledast$, actualmente utilizado en países en desarrollo de América Latina y Asia, contiene una cepa de Salmonella enteritidis muy similar a la de Ratin $®$, por lo que también tendría un riesgo potencial de transmisión y patogenicidad para el ser humano, que no ha sido adecuadamente evaluado. La ausencia de reportes de afección humana asociada con esta nueva formulación de raticida biológico, antes de ser un criterio de inocuidad, puede atribuirse al limitado sistema de vigilancia y capacidad de laboratorio en países en desarrollo.

Las actuales perspectivas del control biológico de roedores plantean lograr la reducción de la fertilidad, antes que incrementar la mortalidad, ya que es muy eficiente la capacidad de estos animales para repoblarse o ser reemplazados por otras colonias de la misma o diferente especie ${ }^{(51)}$. La Organización de las Naciones Unidas para la Agricultura y la Alimentación (FAO) y la OMS ${ }^{(4)}$, estipularon en 1967: Las especies de Salmonella, bajo ninguna circunstancia, deben ser usadas como raticidas. Los roedores rápidamente desarrollan resistencia a serotipos de Salmonella, por lo que este método tiene un valor práctico escaso. Más aun, ha sido demostrado en diferentes países que tales prácticas constituyen un peligro para la salud pública debido a que los serotipos usados son también peligrosos para el ser humano. No conocemos que estas organizaciones hayan modificado esta recomendación.

Más de dos terceras partes de los aislamientos de Salmonella que causan brotes de gastroenteritis asociados con alimentos corresponden a la especie $S$. enteritidis ${ }^{(52)}$. Se ha reportado un incremento de los serotipos de $S$. enteritidis de fagotipos poco frecuentes (diferentes a fa-

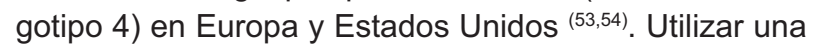
variedad de Salmonella enteritidis para matar roedores no debería ser considerada como una medida de intervención sanitaria por la demostrada capacidad de mutación de las bacterias y su capacidad de adquirir material genético exógeno, al interactuar con cepas patógenas salvajes dentro de los seres vivos o en su entorno. Ante este potencial riesgo y siguiendo las recomendaciones de sus instituciones de salud pública, los países desarrollados y algunos países de Sudamérica como Argentina han prohibido expresamente la producción y comercialización en su territorio de cultivos de enterobacterias para la elaboración de plaguicidas ${ }^{(55)}$. Sin embargo, este producto se sigue comercializando en 12 países de América Central y Sudamérica, incluidos Perú, Bolivia, Ecuador, Cuba, Nicaragua, entre otros, y algunos países de África y Asia (7).
La principal limitación de esta revisión ha sido la escasa información reciente debido a la prohibición de estos raticidas en países desarrollados y la poca publicación de países en desarrollo, por lo que se tuvo que recurrir a búsqueda en la literatura gris, aún no publicada en revistas científicas, mediante motores de búsqueda como Google Scholar. Cuatro de los 12 estudios utilizados, incluido el ensayo clínico, aún no han sido publicados en revistas científicas.

Se concluye que existe evidencia de afección humana, incluso casos fatales, asociada con el uso de raticidas que contienen Salmonella, lo que motivó su retiro del mercado en países desarrollados. La actual versión de raticida biológico utiliza una cepa muy similar y no hay adecuados estudios que garanticen su seguridad en humanos o animales. Se debe realizar estudios controlados por instituciones competentes sin conflicto de interés, que evalúen la transmisibilidad, la patogenicidad y la capacidad de mutación de la variedad de Salmonella enteritidis contenida en las actuales versiones de estos raticidas, antes de su uso en agricultura o en salud pública.

\section{AGRADECIMIENTOS}

A los miembros de UNAGESP que contribuyeron con la revisión del manuscrito: Lely Solari, Edward MezonesHolguín, Paul E. Pachas, Eduardo Falconí, Luz Esther Vásquez y Jorge González.

\section{Financiamiento}

Instituto Nacional de Salud.

\section{Conflictos de Interés}

Los autores declaran no tener ningún conflicto de interés en la elaboración y publicación de este artículo.

\section{REFERENCIAS BIBLIOGRÁFICAS}

1. Friedman CR, Malcolm G, Rigau-Pérez JG, Arámbulo P, Tauxe RV. Public health risk from Salmonella-based rodenticides. Lancet. 1996;347:1705-6.

2. Painter JA, Molbak K, Sonne-Hansen J, Barrett T, Wells JG, Tauxe RV. Salmonella-based rodenticides and public health. Emerg Infect Dis. 2004;10(6):985-87.

3. Threlfall EJ, Ridley AM, Ward LR, Rowe B. Assessment of health risk from Salmonella-based rodenticides. Lancet. 1996;348:616-17.

4. World Health Organization. Joint FAO/WHO. Expert Committee on Zoonoses: third report. Geneva: World Health Organization; 1967. Technical Report Series: 378.

5. Wodzicki K. Prospects for biological control of rodent population. Bull World Health Organ. 1973;48(4):461-67.

6. Healing TD. Salmonella in rodents: a risk to man? CDR (Lond Engl Rev) 1991;1(10):R114-16. 
7. Labotatorios Biológicos Farmacéuticos LABIOFAM Biorat: una nueva alternativa para el control de roedores dañinos [documento en Internet]. La Habana: LABIOFAM; 2009. [Fecha de acceso 25 de junio de 2010]. Disponible en: http://www.labiofam.cu/UserFiles/File/productos/ bioplaguicidas/informativo_biorat.pdf

8. Collazo R, Castro J. Los roedores dañinos: algunos aspectos del control químico y bacteriológico. Rev Inv Pec IVITA (Perú). 1997;8(1):1-9.

9. Tirado MD, Moreno R, Celades ME, Bellido-Blasco J, Pardo FJ. Evolución de los serotipos, fagotipos y resistencia a antimicrobianos de Salmonella sp en el departamento de salud 02 de la provincial de Castellon, España (2000-2006). Rev Chil Infectol. 2009;26(6):520-27.

10. David J. Attribution des cas de salmonelloses humaines aux différentes filières de production animale en France. Adaptabilité et robustesse du modèle bayésien d'attribution par typage microbiologique [thèse doctorat]. Rennes: l'Université Européenne de Bretagne; 2009.

11. Featherstone CA, Reichel R, Snow LC, Davies RH, Christiansen KH, Carrique-Mas JJ, et al. Investigation of risk factors for Salmonella on fattening-turkey farms. Epidemiol Infect. 2010;138(10):1427-38.

12. Garber L, Smeltzer M, Fedorka-Cray P, Ladely S, Ferris K. Salmonella enterica serotype Enteritidis in table egg layer house environments and in mice in USA layer houses and associated risk factors. Avian Dis. 2003; 47(1):134-42.

13. Guard Petter J, Henzler DJ, Rahman MM, Carlson RW. On-farm monitoring of mouse invasive Salmonella enterica serovar Enteritidis and a model for its association with the production of contaminated eggs. Appl Environ Microbiol. 1997;63(4):1588-93.

14. Henzler DJ, Opitz HM. Role of rodents in the epidemiology of Salmonella enterica serovar Enteritidis and other Salmonella serovars in poultry farms. En: Saeed AM (ed). Salmonella enteric serovar enteritidis in humans and animals: epidemiology, pathogenesis and control. lowa: lowa State University Press, 1999. p. 331-40.

15. Hilton AC, Willis RJ, Hickie $\mathbf{S}$. Isolation of Salmonella from urban wild brown rats (Rattus norvegicus) in the West Midlands. Int J Environ Health Res. 2002; 12 (2): 163-8.

16. Lapuz R, Tani H, Sasai K, Shirota K, Katoh H, Baba E. The role of roof rats (Rattus rattus) in the spread of Salmonella enteritidis and $S$. infantis contamination in layer farms in eastern Japan. Epidemiol Infect. 2008;136(9): 1235-43.

17. León V, Fraschina J, Busch M. Rodent control at different spatial scales on poultry farms in the province of Buenos Aires, Argentina. Int Biodeterior Biodegradation. 2009; 63(8):1113-18.

18. Leslie PH. The bacteriological classification of the principal cultures used in rat and mouse control in Great Britain. J Hyg (Lond). 1942;42(5):552-62.

19. Meerburg BG, Brom FWA, Kijlstra A. The ethics of rodent control. Pest Mnag Sci. 2008;64(12):1205-11.

20. Meerburg BG, Kijlstra A. Role of rodents in transmission of Salmonella and Campylobacter. J Sci Food Agriculture. 2007;87(15):2774-81.

21. Meerburg PJ. Sporadic cases of Salmonellosis associated with mice in a home for aged persons. J Appl Bacteriol. 1982;53: xiv.
22. Rabsch W, Tschape H, Baumler AJ. Nontyphoidal salmonellosis: emerging problems. Microbes Infect. 2001;3(3):237-47.

23. Singleton GR, Brown PR, Jacob J. Ecologically-based rodent management: its effectiveness in cropping systems in South-East Asia. NJAS-Wageningen $\mathrm{J}$ Life Science. 2004;52(2): 163-71.

24. Singleton GR. The prospects and associated challenges for the biological control of rodents. In: Procedings of the $16^{\text {th }}$ Vertebrate Pest Conference. Lincoln: University of Nebraska; 1994. p.301-7.

25. Velge P, Cloeckaert A, Barrow P. Emergence of Salmonella epidemics: the problems related to Salmonella enterica serotype Enteritidis and multiple antibiotic resistance in other major serotypes. Vet Res. 2005;36(3):267-88.

26. Villafaña F, de Armas R, Montero G, Díaz M. Efectividad en el uso de rodenticida biológico Biorat $₫$ en comparación con el rodenticidaquímico parael control de los roedores sinantrópicos en objetivos urbanos de la provincia de Cienfuegos, Cuba. Bol Malariol Salud Ambient. 2000;40(1):3-8.

27. Villafaña F, Montero G, Díaz M, Bornote J. Efectividad del rodenticida Salmocumarín en objetivos pecuarios y urbano. Rev Cubana Med Trop. 1995;47(2):83-87.

28. Villafaña F, Silva M, Ruiz J, Sánchez L, Campos A. Evaluación del impacto del biorrodenticida Biorat ${ }^{\circledR}$ en poblaciones de roedores establecidos en varios cultivos en la República de Costa Rica. Rev Cubana Med Trop. 1999;51:185-88.

29. Ibarra $X$, Hernández CM. Evaluación de la seguridad y eficacia de BIORAT por laboratorios de Investigación en América Central. En: LABIOFAM Enterprise Group. Dossier Biorat: analytic inform. La Habana: LABIOFAM Enterprise Group; 2009. p. 55-57.

30. Instituto de Nutrición e Higiene de los Alimentos. Respuesta a interrogante del Biorat [página en Internet]. La Habana: INHA; 2003. [Fecha de acceso: 25 de junio de 2010]. Disponible en: http://www.inha.sld.cu/ vicedirecciones/Biorat.htm.

31. Rodríguez CT, De la Vega Lugo J. Inocuidad de Sallmonella enteritidis var danysz, lisina negativa, fagotipo 6a aplicado a los paneles de abeja (Aphis mellifera). En: LABIOFAM Enterprise Group. Dossier Biorat: analytic inform. La Habana: LABIOFAM Enterprise Group; 2009. p. 89-91.

32. Labotatorios Biológicos Farmacéuticos LABIOFAM. Safety of Salmonella enteritidis var danysz lisina negativa for domestic animals. Dogs, sheep, swine. En: LABIOFAM Enterprise Group. Dossier Biorat: analytic inform. La Habana: LABIOFAM Enterprise Group; 2009. p. 53-54.

33. Gracie MA. The epidemiology of Salmonella infections in man: use of bacterial rodenticides. Perspect Public Health. 1957;77(4):179-80.

34. Negrin O, Tejada M, Lewis F. Uso de Salmonella enteriditis para el control de roedores en caña de azúcar. Ciencia y Técnica en la Agricultura Protección de Plantas (Cuba). 1989; 12: 89-96.

35. World Health Organization. Technical discussions: Zoonoses. WHO Chron. 1954;8 226.

36. Villafaña F, Espino R, Montero G, Bornote JG, Diaz M, et al. Efectividad del rodentencida Biorat ${ }^{\circ}$ en ambientes pecuarios y urbanos. Rev Patol Trop. 1995;24:11-19. 
37. Faulde MK. Rats and mice. Neglected vectors and reservoirs of dangerous infectious diseases? Hyg Medizin. 2004;29(6):206-16

38. Ponomarev BP. [The preparation and application of bactocoumarin for rat control]. Veterinariia. 1966;43(11): 90-91. [artículo en Ruso]

39. Rybin AP. [Use of bactorodencide in rodent control]. Veterinariia; 1977;(3):52-53. [artículo en Ruso]

40. Bravo JR, Díaz H, Bravo L, Ramírez AM, Monté R, Martínez G, et al. Evaluación de la seguridad para humanos de la ingestión de Salmonella enteritidis, var. danysz, lysine negativa, phage tipo 6a. En: LABIOFAM Enterprise Group. Dossier Biorat: analytic inform. La Habana: LABIOFAM Enterprise Group; 2009. p. 181-223.

41. Kristensen M. Observation on Salmonella enteritidis var Danysz and Salmonella enteritidis var Chaco in cases of disease in man. Acta Pathol Microbiol Scand. 1946;23(4):299-316.

42. Spray RS. An outbreak of food poisoning probably due to rat virus. JAMA. 1924;86(2):109-11.

43. Taylor J. Bacterial rodenticides and infection with Salmonella enteritidis. Lancet. 1956; 270: 630-3.

44. Hernández N, Torres A, Ramírez M, García I, Menéndez Z, Castro J. Comportamiento de peces dulceacuícolas en contacto con Salmonella enteritidis var. danysz, La Habana Cuba. Rev Peru Biol. 1999;6(2):131-36.

45. Labotatorios Biológicos Farmacéuticos LABIOFAM Salmonella enteritidis var. Danysz lysine negative phage type 6a.Acute oral toxicity in rabbits. En: LABIOFAM Enterprise Group. Dossier Biorat: analytic inform. La Habana: LABIOFAM Enterprise Group; 2009. p. 144-51.

46. Rejo T, Masden V, Tamargo B, Gutierrez J, Viamontes $\mathbf{O}$, Navarro $\mathbf{N}$, et al. Results and considerations about serologic test in fowl infected by drinking water with cultures of Salmonella enteritidis var, danysz lisina negativa pafo tipo 6A. En: LABIOFAM Enterprise Group. Dossier Biorat: analytic inform. La Habana: LABIOFAM Enterprise Group; 2009. p. 92-98.

47. Rodríguez N, Icochea $\mathbf{E}$, Calle S, Noé N. Estudio de inocuidad de Salmonella enterica, subespecie enterica, serotipo enteritidis, var. danysz, lisina negativa en pollos parrilleros. Rev Inv Vet Peru. 2006;17(1):33-38.
48. Leyva V, Espino R, González R. Estudio microbiológico para la exoneración del biorat como contaminante de camarones cosechados en Nicaragua. Alimentaria 2003;40(344):23-27.

49. Joya JRE, Quevedo E, Ponce JL, Reyes JM, Balaña $\mathbf{P}$, Solares S. Efectividad del rodenticida biológico Biorat para el control de roedores plaga en Escuintla. En: Libro de resúmenes del VI Congreso de la Asociación de Técnicos Azucareros de Latinoamerica y el Caribe (ATALAC). Guayaquil: ATALAC; 2006. p. 185-88.

50. Tenover FC, Arbeit RD, Goering RV. How to select and interpret molecular strain typing methods for epidemiological studies of bacterial infections: a review for healthcare epidemiologists. Infect Control Hosp Epidemiol. 1997;18(6):426-39.

51. Hardy CM. Current status of virally vectored immunocontraception for biological control of mice. Soc Reprod Fertil Suppl. 2007;63:495-506.

52. Galanis E, Lo Fo Wong DM, Patrick ME, Binsztein N, Cieslik A, Chalermchikit T, et al. Web-based surveillance and global Salmonella distribution, 2000-2002. Emerg Infect Dis. 2006; 12: 381-8.

53. Lynch M, Painter J, Woodruff R, Braden C, Centers for Disease Control and Prevention. Surveillance for foodborne-disease outbreaks--United States, 1998-2002. MMWR Surveill Summ. 2006;55(10):1-42.

54. Gormley FJ, Little CL, Rawal N, Gillespie IA, Lebaigue S, Adak GK. A 17-year review of foodborne outbreaks: describing the continuing decline in England and Wales (1992-2008). Epidemiol Infect. 2010 Aug 9:1-12. [Epub ahead of print]

55. Argentina, Administración Nacional de Medicamentos, Alimentos y Tecnología Médica. Disposición 3.316/2005. Prohíbase el empleo de cultivos de enterobacterias en la formulación de productos plaguicidas domisanitarios. Buenos Aires: ANMAT; 2005.

Correspondencia: Alberto Mendoza.

Dirección: Cápac Yupanqui 1400, Lima 11, Perú.

Teléfono: (511) 617-6200 Anexo: 2143

Correo electrónico: cmendoza@ins.gob.pe 